\title{
PAPERS
}

\section{Role of apoptosis induced by Helicobacter pylori infection in the development of duodenal ulcer}

\author{
K Kohda, K Tanaka, Y Aiba, M Yasuda, T Miwa, Y Koga
}

\begin{abstract}
Background-Helicobacter pylori affects gastric epithelium integrity by acceleration of apoptosis. However, it remains unclear what product of the bacteria causes apoptosis, or whether or not the apoptosis is involved in the development of ulcers.
\end{abstract}

Aims-To elucidate the factor from $H$ pylori that causes acceleration of apoptosis and the role of apoptosis in the development of duodenal ulcer in $\mathrm{H}$ pylori infection.

Patients-Five $H$ pylori negative healthy volunteers, $47 \mathrm{H}$ pylori positive patients with duodenal ulcer, and $35 \mathrm{H}$ pylori positive patients with gastric ulcer.

Methods-An endoscopic examination was carried out to diagnose ulcers and determine their clinical stage. To analyse apoptosis, a cell cycle analysis was performed using biopsy specimens.

Results-There was a significant correlation between the urease activity of the $H$ pylori strain and the level of apoptosis induced by this bacterial strain. Moreover, in duodenal ulcer patients infected with $H$ pylori, the patients with an active ulcer exhibited a significantly higher level of apoptosis than those with ulcers at both the healing and scarring stages.

Conclusion-These findings suggest that acceleration of apoptosis in the antral mucosa caused by the urease of $H$ pylori plays a crucial role in the development of ulcers in the duodenum.

(Gut 1999;44:456-462)

Keywords: apoptosis; Helicobacter pylori; urease; duodenal ulcer; gastric ulcer

Helicobacter pylori has been shown to play an important role in the pathogenesis of duodenal ulcer (DU) disease. ${ }^{1}$ However, the mechanism by which $H$ pylori infection, which predominantly affects the gastric mucosa, induces ulceration of the duodenum remains to be elucidated. Many studies have indicated that $H$ pylori impairs the gastric epithelial layer, either by a variety of cytotoxins produced by the bacteria or as a result of the accompanying inflammatory response by the release of various cytokines and cytotoxic endogenous products. ${ }^{2}$
However, the predominant location of $H$ pylori infection in the gastric mucosa in patients with DU diseases also strongly suggests the existence of some mechanism other than those mentioned above. One possible explanation is that the $H$ pylori infection in the gastric antrum blocks the normal physiological inhibitory mechanism of the antrum to both gastrin- and acid-releasing cells, resulting in increased gastrin release as well as impaired inhibition of gastric acid secretion, leading to development of DU disease. ${ }^{3}{ }^{4}$

Apoptosis has been shown to occur in tissues undergoing such diverse processes as embryogenesis, metamorphosis, and immunological development. ${ }^{5}$ Apoptosis is a physiological suicide mechanism which maintains homoeostasis, in which cell death naturally occurs during tissue turnover. In the gastric epithelium, apoptosis also seems to play an essential role in maintaining tissue integrity, and the rate of new cell production by proliferation is matched by the rate of cell loss by apoptosis. ${ }^{6}$ Moss and colleagues ${ }^{7}$ reported that the number of apoptotic cells in the gastric epithelium increases with $H$ pylori infection and decreases after eradication of the bacterium.

The mechanism by which $H$ pylori infection induces apoptosis has yet to be elucidated, but the ammonia generated from the breakdown of urea by $H$ pylori urease may be the virulent factor. Tsuji and colleagues ${ }^{8}$ have shown that the long term administration of ammonia induces mucosal atrophy in the antrum, suggesting increased cell loss by apoptosis in this region of the stomach. These results led to the hypothesis that the acceleration of apoptosis by $H$ pylori infection in the antrum may disrupt the normal physiological regulatory mechanism of gastrin and/or acid production exerted by the antrum, resulting in the development of DU disease. To test this hypothesis, we investigated the relation between apoptosis and the development of DU in association with $H$ pylori infection in humans by examining clinical gastric specimens and properties, including urease activity, of $H$ pylori strains isolated from them.

Abbreviations used in this paper: DU, duodenal ulcer; GU, gastric ulcer; TUNEL, terminal uridine deoxynucleotide nick end labelling; EGF, epidermal growth factor. 
Table 1 Subject profile

\begin{tabular}{lllll}
\hline & $n$ & Mean age (range) & Sex $(M / F)$ & Apoptosis (\%)* \\
\hline $\begin{array}{l}\text { Control } \\
\text { Duodenal ulcer }\end{array}$ & 5 & $30(28-33)$ & $4 / 1$ & $7.1(2.0)$ \\
$\quad$ Active & 15 & $49(18-66)$ & $12 / 3$ & \\
$\quad$ Healing & 17 & $47(29-61)$ & $13 / 4$ & \\
$\quad$ Scarring & 15 & $50(41-69)$ & $9 / 6$ & \\
$\quad$ Total & 47 & & $34 / 13$ & $20.2(1.5)$ \\
Gastric ulcer & & $51(32-69)$ & $15 / 6$ & \\
$\quad$ Active & 21 & $51(40-67)$ & $6 / 1$ & \\
Healing & 7 & $52(43-72)$ & $5 / 2$ & \\
$\quad$ Scarring & 7 & & $26 / 9$ & $19.1(1.6)$ \\
Total & 35 & & &
\end{tabular}

${ }^{\star}$ Expressed as mean (SEM).

\section{Methods}

PATIENTS AND STUDY DESIGN

Table 1 shows the mean age, age range, and gender of the subjects examined in this study including five $H$ pylori negative healthy volunteers, $47 H$ pylori positive patients with DU disease, and $35 \mathrm{H}$ pylori positive patients with gastric ulcer (GU) disease.

Endoscopic examination was used to diagnose the diseases and determine their stages. The ulcer stage was classified as active, healing, or scarring according to the classification of Sakita and colleagues ${ }^{9}$. Briefly, these stages are defined as follows: active, the surrounding mucosa is oedematously swollen and little regenerating epithelium is seen endoscopically; healing, the white coating becomes thin, and the regenerating epithelium extends into the ulcer; scarring, the regenerating epithelium completely covers the floor of the ulcer. Biopsy specimens were collected from the gastric antrum and the ulcer margin in cases of DU and GU disease respectively. In the case of GU disease, biopsy sampling from the antrum was not performed because it became difficult if the ulcer was located on the antral region. Infection with $H$ pylori was confirmed by the rapid urease test on biopsy samples, and the number of $H$ pylori colonising the sample was determined by using Skirrow agar plates as previously reported. ${ }^{10}$ Some of the $H$ pylori positive subjects including eight with DU (four active and four healing) and 10 with GU (six active and four healing) received a two week course of eradication therapy. The following regimen was used: $20 \mathrm{mg}$ omeprazole/day, $1000 \mathrm{mg}$ amoxicillin/day, and $400 \mathrm{mg}$ clarithromycin/day. Just before and three months after this therapy, an endoscopic examination was performed to confirm that the ulcer was healed and also to collect biopsy samples for bacteriological examination. Eradication of infection by antibiotic therapy was defined as successful when both the rapid urease test of the biopsy sample and the bacterial culture of the sample using Skirrow plates were negative.

\section{ANALYSIS OF APOPTOSIS}

Apoptosis was investigated by cell cycle analysis using flow cytometry. ${ }^{11}$ Briefly, single gastric epithelial cells were fixed with $2 \%$ paraformaldehyde, permeabilised with $0.1 \%$ Nonidet $\mathrm{P} 40$, and treated with $0.05 \mathrm{mg} / \mathrm{ml} \mathrm{RNase} A$ for 30 minutes at $37^{\circ} \mathrm{C}$. After being washed, the cells were stained with propidium iodide and then analysed using a flow cytometer. The cells in a discrete subpopulation of signals under the $\mathrm{G}_{0} / \mathrm{G}_{1}$ cell cycle region (subdiploid cells) were designated as undergoing apoptosis. Single gastric epithelial cells were prepared from biopsy specimens by the method of Kobayashi and colleagues. ${ }^{12}$ In brief, the gastric tissue specimens were minced into pieces and stirred into Hanks balanced salt solution containing $0.1 \%(\mathrm{w} / \mathrm{v})$ collagenase A (Boehringer Mannheim $\mathrm{GmbH}$, Mannheim, Germany) for 60 minutes at room temperature. The tissue suspension was then filtered through a cotton gauze, and the filtrate was centrifuged at $750 \mathrm{~g}$ for five minutes to harvest single cells.

Examination of apoptosis in situ using the method of terminal uridine deoxynucleotide nick end labelling (TUNEL) histochemistry was performed as described by Gavrieli et al. ${ }^{13}$ Briefly, 4-6 $\mu \mathrm{m}$ sections were cut from paraffin embedded tissue blocks and mounted on glass slides. The sections were deparaffinised through xylene and alcohol, washed, and then digested with proteinase $\mathrm{K} 20 \mu \mathrm{g} / \mathrm{ml}$ for 15 minutes at room temperature, followed by washing and blocking of endogenous peroxidase with $2 \%$ hydrogen peroxide for five minutes.

After preincubation with terminal transferase reaction buffer containing $200 \mathrm{mM}$ potassium cacodylate, $25 \mathrm{mM}$ Tris/ $\mathrm{HCl}, \mathrm{pH}$ 6.6 , and $0.25 \mathrm{mg} / \mathrm{ml}$ bovine serum albumin (Boehringer Mannheim) for 10 minutes, the sections were incubated at $37^{\circ} \mathrm{C}$ for 90 minutes with the same buffer containing, in addition, 1 $\mathrm{mM}$ cobalt chloride, terminal transferase $(0.5$ $\mathrm{U} / \mu \mathrm{l})$, and $0.4 \mu \mathrm{M}$ digoxigenin-11deoxyuridine triphosphate (digoxigenin-11dUTP). The reaction was terminated by the addition of a solution containing $300 \mathrm{mM}$ $\mathrm{NaCl}$ and $30 \mathrm{mM}$ sodium citrate, and the slides were washed in water. Incorporated digoxigenin-11-dUTP was detected by incubation with peroxidase conjugated $\mathrm{Fab}$ fragments of anti-digoxigenin (Boehringer Mannheim) at a concentration of $1: 300$ in $100 \mathrm{mM}$ Tris/ $\mathrm{HCl}$ buffer, $\mathrm{pH} 7.5$, containing $150 \mathrm{mM} \mathrm{NaCl}$ at room temperature for 30 minutes. The slides were then washed in water, counterstained with methyl green, and mounted.

\section{FLOW CYTOMETRY}

To analyse the surface cell marker, single gastric epithelial cells were washed once with Hanks balanced salt solution and then stained with anti-epidermal growth factor (EGF) receptor mouse monoclonal antibody (clone $6 \mathrm{~F} 1$; Immunotech, Marseille, France) according to the manufacturer's instructions. Bound antibodies were disclosed with fluorescein isothiocyanate conjugated anti-mouse IgG antibody (Tago Inc, Burlingame, California, USA). The machine was gated to include single cells but to exclude any cell debris and clumps of cells according to forward and side scatter patterns.

\section{CHARACTERISATION OF H PYLORI STRAINS}

$H$ pylori was isolated from the gastric biopsy materials. The wet weight of the biopsy material was measured before the procedure in 
order to determine bacterial number. The bacteria were grown on Skirrow plates, so that the number of colony forming units in the biopsy material (number/g wet tissue) could be counted, and then inoculated into a Brucella broth containing $5 \%$ fetal calf serum in a microaerophilic atmosphere at $37^{\circ} \mathrm{C}$ to prepare a fresh sample of bacteria as previously described. ${ }^{10}$ To determine the urease activity of $H$ pylori, freshly prepared $H$ pylori was suspended at $1 \times 10^{9}$ colony forming units $/ \mathrm{ml}$ in phosphate buffered saline. Then $0.1 \mathrm{ml}$ of this bacterial solution was mixed with $0.9 \mathrm{ml}$ of a urease substrate solution $(50 \mathrm{mM}$ urea in 100 $\mathrm{mM}$ acetate buffer), incubated at $37^{\circ} \mathrm{C}$ for 20 minutes, and then applied to an AmmoniaTest-Wako Kit (Wako, Osaka, Japan) to measure the ammonia concentration according to the manufacturer's instructions. The amount of protein was measured using a Bio-Rad Protein Assay Kit (Bio-Rad Laboratories, Hercules, California, USA). In a preliminary experiment, the amount of protein in a bacterial solution correlated closely with the colony forming units contained in the solution so that the urease activity per protein can also be considered as the urease activity per bacterial body. To determine the vacuolating cytotoxin activity, $1 \times 10^{7}$ colony forming units of $H$ pylor $i$ were inoculated into $10 \mathrm{ml}$ Brucella broth containing $5 \%$ fetal calf serum and then incubated for seven days as described above. The culture was then diluted serially in Dulbecco's modified Eagle's medium containing $10 \%$ fetal calf serum and $10 \mathrm{mM}$ ammonium chloride before being added to RK13 cells. ${ }^{1314}$ The maximum dilution at which more than $80 \%$ of RK13 cells become vacuolated was regarded as positive and this value was recorded.

HISTOLOGICAL ANALYSIS

Sections from biopsy samples were stained with haematoxylin and eosin. Multiple high power fields were examined by one experienced pathologist blinded to the patient's clinical diagnosis. The histological features of inflammation and epithelial damage were graded from 1 to 3 for each biopsy specimen. The scored features included lymphocyte infiltration, neutrophil infiltration, intestinal metaplasia, and epithelial erosion. Lymphocyte infiltration was graded as follows: 0, lymphocytes present in normal numbers; 1 , a minor and equivocal increase in the lymphocyte number; 2 , a moderate increase in the lymphocyte number; 3 , a maximal or submaximal increase in the lymphocyte number in most of the section. Neutrophil infiltration was graded as follows: 0, neutrophils difficult to find; 1 , a minor increase in neutrophils but they were not in groups and did not invade the gland necks; 2, the neutrophils definitely increased, forming groups or invading the gland necks; 3 , groups of neutrophils were visible in nearly every field. Intestinal metaplasia and epithelial erosion were graded as follows: 0 , not found; 1 , found.
STATISTICAL ANALYSIS

Statistical analysis was by Student's $t$ test, the paired $t$ test, and the scattergram plot. Histological scores were non-parametric and were therefore analysed by the Mann-Whitney $U$ test. Numerical data in the text represent the mean (SEM) except where indicated otherwise.

\section{Results}

CHARACTERISATION OF CELLS WITH RESPECT TO APOPTOSIS

Apoptotic cells were determined by cell cycle analysis of the single cells obtained from gastric

$A$
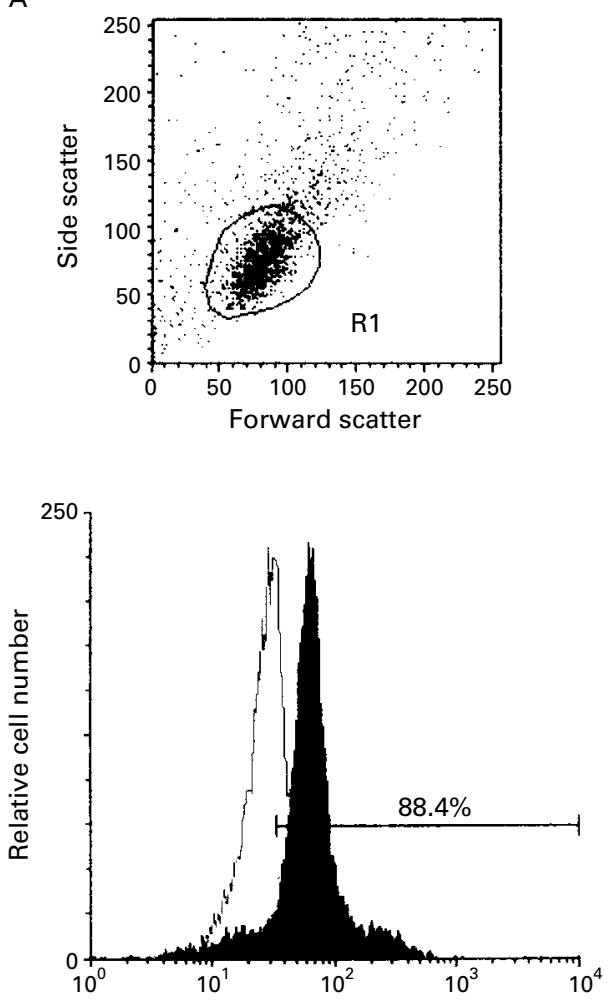

B

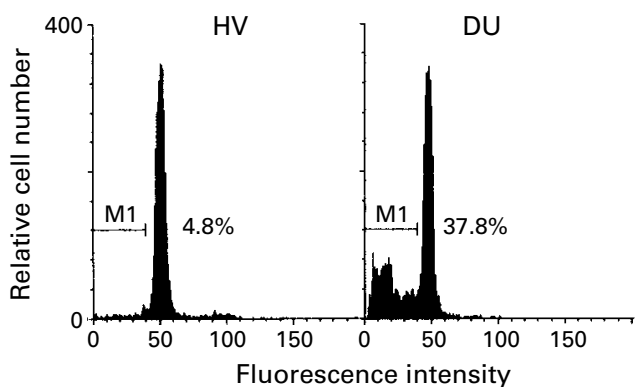

Figure 1 Flow cytometry analysis of gastric mucosal cells. (A) The flow cytometer was gated to include single cells but to exclude any cell debris and clumps of cells according to the forward and side scatter patterns (upper panel). Single gastric mucosal cells were stained with mouse anti-epidermal growth factor receptor antibody and fluorescein isothiocyanate conjugated anti-mouse IgG antibody (lower panel, closed configuration) or fluorescein isothiocyanate conjugated anti-mouse IgG antibody only (lower panel, open configuration). (B) Representative data of the cell cycle analysis using specimens from healthy volunteers $(H V)$ and patients with duodenal ulcer (DU) are shown, and the percentage of apoptosis cells in these specimens is also indicated. 

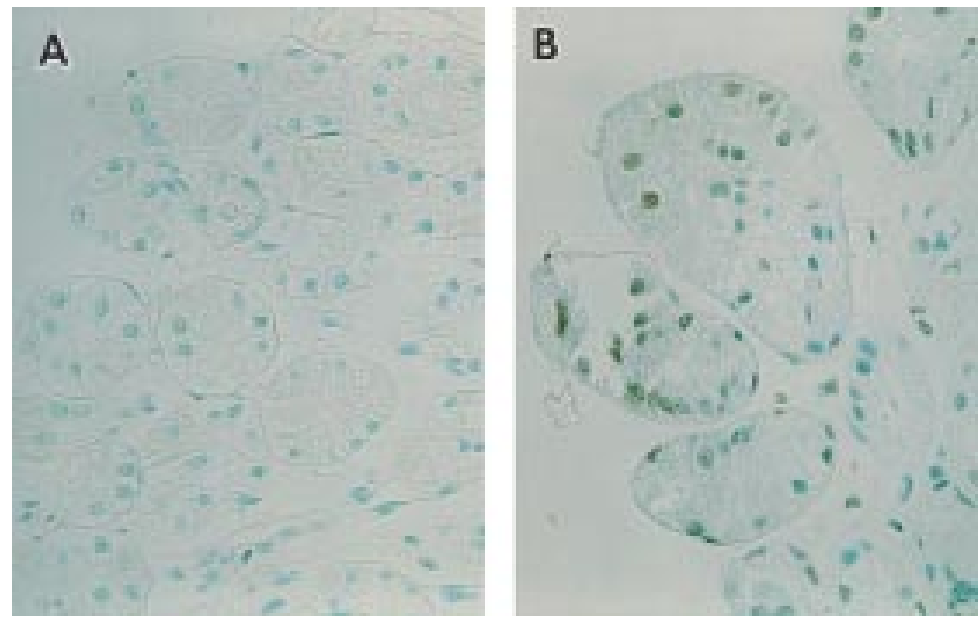

Figure 2 Gastric antral biopsy samples obtained from a healthy volunteer $(A)$ and a patient with a duodenal ulcer $(B)$ were examined by TUNEL histochemistry.

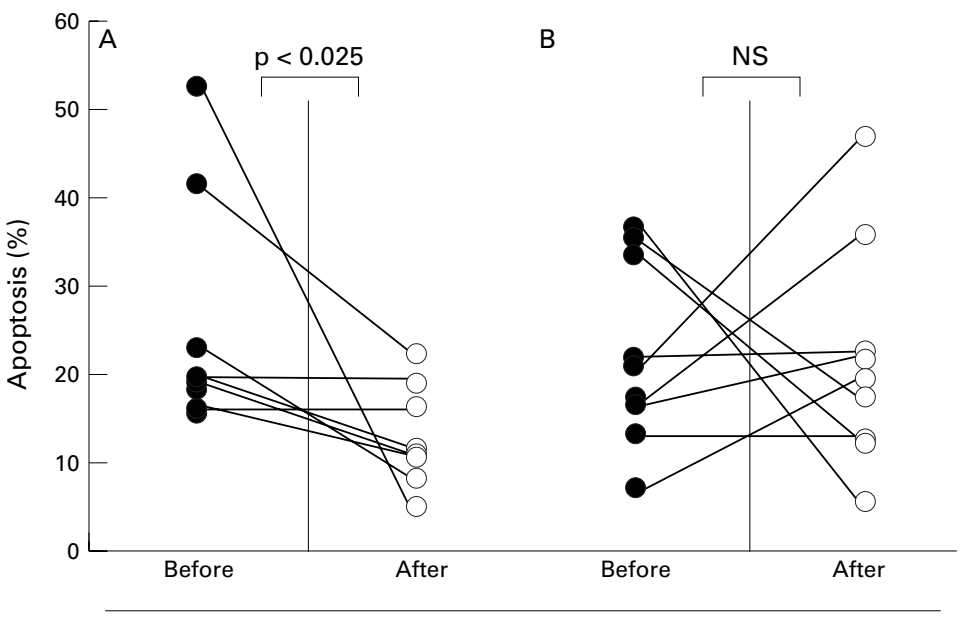

Eradication of $\mathrm{H}$ pylori

Figure 3 Effect of the eradication of $H$ pylori on the level of apoptosis. Gastric biopsy samples were taken from $(A)$ patients with $D U$ and $(B)$ those with $G U$ before the administration of antibiotics and three months after eradication therapy. The level of apoptosis in these samples was evaluated by flow cytometric analysis. A statistical analysis was performed using the paired t test.

biopsy samples. The cells to be examined were gated by scatter analysis as indicated in the upper panel of fig 1A. Around $90 \%$ of these cells expressed EGF receptor, which is the hallmark of epithelial cells. The lower panel of fig $1 \mathrm{~A}$ shows a representative flow cytometry pattern of this surface cell maker. As shown in fig $1 \mathrm{~B}$, the cells in a subpopulation under the $\mathrm{G}_{0} / \mathrm{G}_{1}$ cell cycle region as indicated by a horizontal bar were regarded as apoptotic, and the percentage of these apoptotic cells in all the cells is shown.

When the biopsy samples were examined by TUNEL histochemistry, a considerable number of epithelial cells in the sample from a patient with DU (fig 2B) were also stained positively by TUNEL, while a few in the sample from a healthy volunteer were not stained (fig 2A).

By using cell cycle analysis, the level of apoptosis in the biopsy samples from the healthy volunteers and ulcer patients was examined in the following experiments. The percentage of apoptotic cells was significantly lower in healthy volunteers $(7.1(2.0) ; \mathrm{n}=5)$
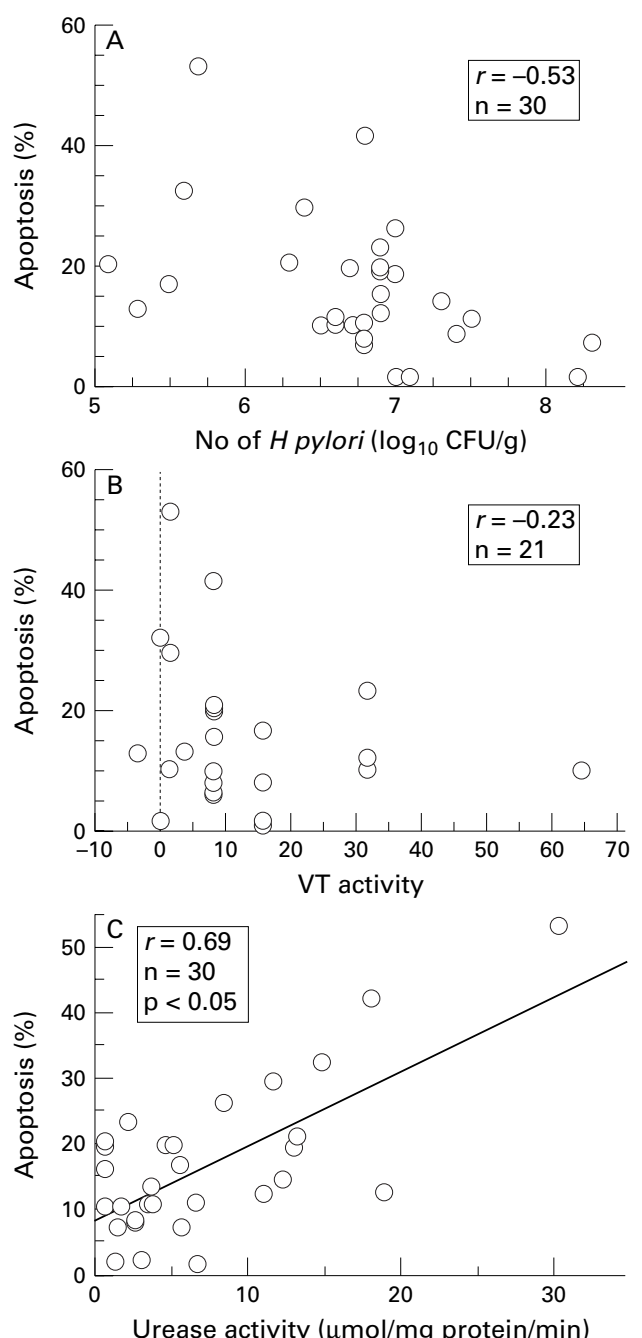

Figure 4 Relation between apoptosis and the propeties of $H$ pylori. The relation between the level of apoptosis in mucosal cells examined by flow cytometry and the bacteriological properties of $H$ pylori including the number of bacteria in the mucosal tissue $(A)$, the activity of the vacuolating toxin (VT) in the culture supernatant of bacteria $(B)$, and the urease activity of the bacteria $(C)$ were plotted. The relations between these factors were then statistically analysed by a scattergram plot.

than in patients with DU (20.2 (1.5) ( $\mathrm{n}=47)$; $\mathrm{p}<0.01)$ or $\mathrm{GU}(19.1(1.6)(\mathrm{n}=35) ; \mathrm{p}<0.05)$ (table 1). All of the healthy volunteers were $H$ pylori negative, whereas the subjects with DU and GU were $H$ pylori positive, suggesting that infection of the stomach with $H$ pylori was responsible for the acceleration of apoptosis of the epithelial cells in these patients. Complete eradication of $H$ pylori by administration of antibiotics significantly lowered the level of apoptosis in DU patients (26.1 (4.9) $v 13.1$ (2.1) $(\mathrm{n}=8) ; \mathrm{p}<0.025$; fig 3$)$. In GU patients, on the other hand, eradication of $H$ pylori did not decrease the level of apoptosis $(21.8$ (3.2) $v$ $21.8(3.8)(\mathrm{n}=10) ; \mathrm{p}>0.1)$. Therefore colonisation by $H$ pylori of the stomach, including the antrum region, is closely related to acceleration of apoptosis in the antral mucosa of patients with DU, but infection with $H$ pylori is not directly related to the increased apoptosis in the gastric mucosa of patients with GU. 


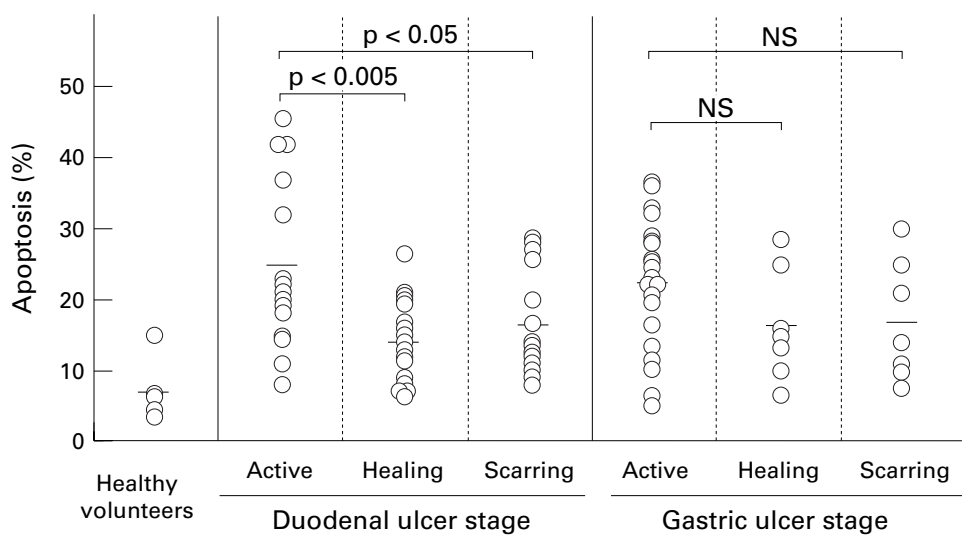

Figure 5 Level of apoptosis at each clinical stage in ulcer disease. The level of apoptosis was examined by flow cytometry using gastric biopsy samples obtained from healthy volunteers and patients with duodenal ulcer at the active, healing, or scarring stage, and patients with gastric ulcer at the active, healing, or scarring stage. Statistical analysis was by Student's $t$ test. $\mathrm{p}<0.05$ ) (fig $4 \mathrm{C}$ ). The other variables showed no significant correlation with the level of apoptosis (fig $4 \mathrm{~A}$ and $\mathrm{B}$ ). It was therefore assumed that the product generated by urease, possibly ammonia, increases apoptosis in the antral mucosa on infection with $H$ pylori.

\section{APOPTOSIS AT EACH STAGE OF DU AND GU}

DISEASE

To elucidate the role of apoptosis in the development of ulceration in the duodenum and stomach, the degree of apoptosis was examined at each stage of DU and GU disease (fig 5). In the DU patients, the patients at the active stage exhibited a significantly higher level of apoptosis than those at either the healing stage $(24.8$ $(3.1)(\mathrm{n}=15) v 14.3(1.4)(\mathrm{n}=17) ; \mathrm{p}<0.005)$ or scarring stage (24.8 (3.1) $(\mathrm{n}=15) v 16.3$ (1.9) $(\mathrm{n}=15) ; \mathrm{p}<0.05)$. In GU patients, however, no significant difference in the degree of apoptosis was found in the active (22.4 (1.9); n $=21)$, healing (16.4 (3.0); $\mathrm{n}=7)$, and scarring (17.1 (3.2); $\mathrm{n}=7$ ) stages. Apoptosis in the antral mucosa was therefore suggested to be closely associated with the development of ulceration in the duodenum, but not in the stomach.

To investigate whether or not the degree of inflammation is associated with the stage in DU, biopsy samples from DU patients at each stage were examined for histological features including lymphocyte infiltration, neutrophil infiltration, intestinal metaplasia, and epithelial erosion (fig 6). Although the specimens of the active, healing, and scarring stages of DU all exhibited a higher grade with respect to apoptosis induced in the biopsy specimens colonised by this strain $(r=0.69, \mathrm{n}=30$,

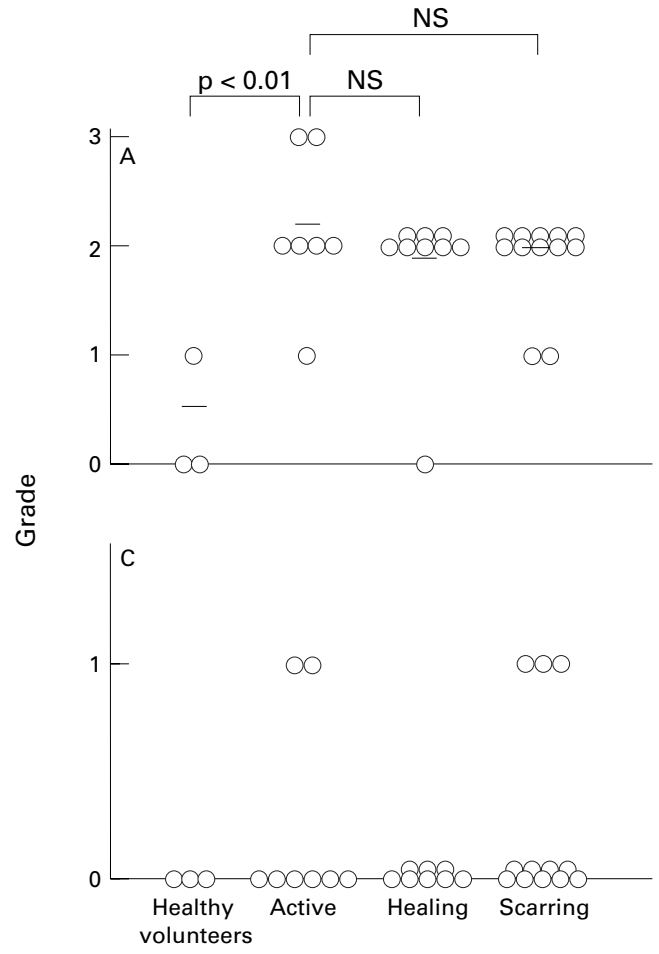

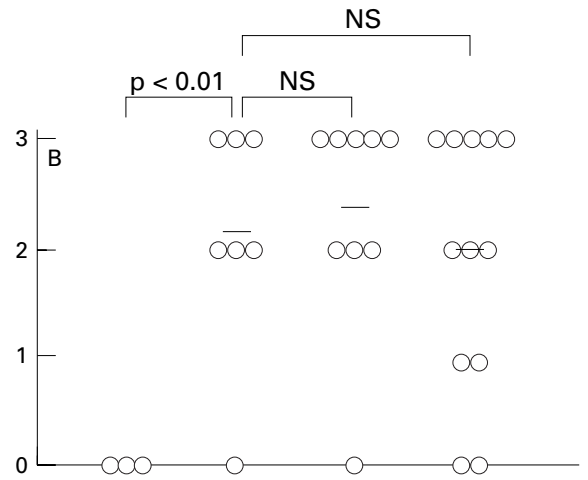

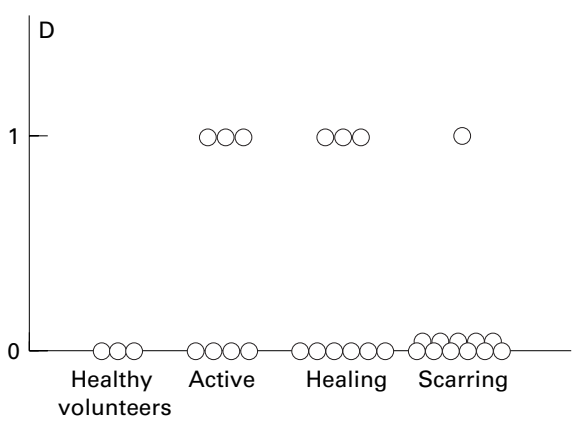

Duodenal ulcer stage

Figure 6 Histological analysis of gastric biopsy specimens. Histological features including lymphocyte infiltration (A), neutrophil infiltration (B), intestinal metaplasia (C), and epithelial erosion (D) were examined using gastric biopsy samples from healthy volunteers and patients with active, healing, and scarring duodenal ulcers, and their grades determined. Statistical analysis was by the Mann-Whitney U test. 


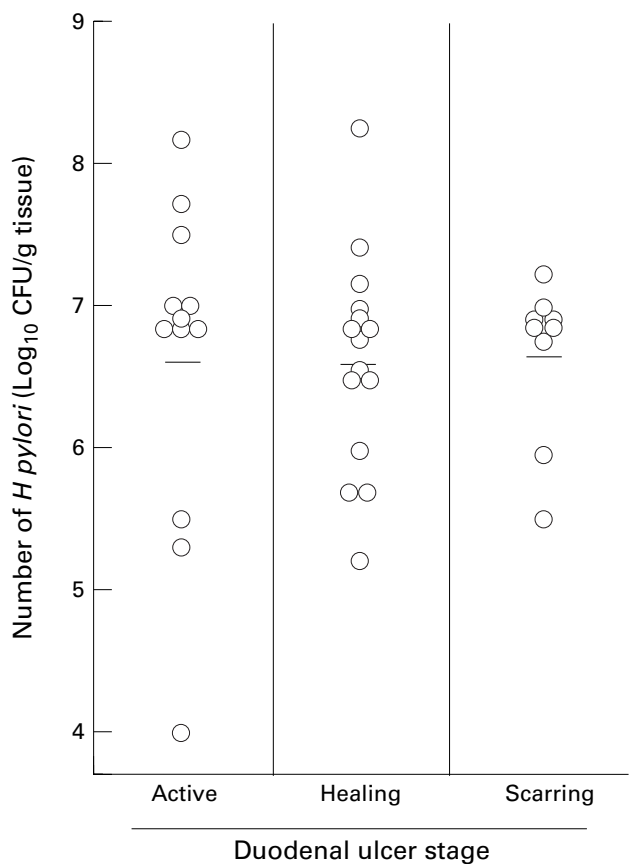

Figure 7 Number of H pylori in the antral mucosa of patients with duodenal ulcer at different clinical stages. Antral biopsy samples were taken from patients with active, healing, and scarring ulcers, and the number of $H$ pylori colonising the sample was determined.

lymphocyte infiltration (fig 6A) and neutrophil infiltration (fig 6B) than did those from healthy volunteers, no significant difference in the grade was found between the specimens, implying that the inflammatory events disclosed by histological analysis of the antral mucosa are not relevant to the development of ulceration in the duodenum. These findings suggest that the events that induce apoptosis, which are associated with the development of duodenal ulceration, take place independently of the events that cause inflammation.

To determine whether the size of the bacterial load in the antral mucosa is associated with the development of ulceration in the duodenum, the number of $H$ pylori in the antral mucosa was measured using biopsy samples from each stage of DU. As shown in fig 7, the number of bacteria was about the same in the three stages of ulceration, suggesting that the size of the bacterial load is not related to the stage of ulceration in the duodenum once colonisation by $H$ pylori has been established in the stomach.

Taken together, the results suggest that apoptosis correlates closely with the stage of DU, and therefore appears to be a crucial pathological event leading to the development of ulceration.

\section{Discussion}

Apoptosis is an essential part of the cycle of cellular turnover in many tissues, including the gastrointestinal tract. Moss and colleagues ${ }^{7}$ showed $H$ pylori infection to be associated with increased epithelial apoptosis in the gastric mucosa, and that, after eradication of $H$ pylori, apoptosis decreases to the levels found in normal controls. In line with their report, we also found an increase in apoptosis in epithelial cells of the antral mucosa in $H$ pylori infected DU patients as well as a decrease in apoptosis after eradication of this bacterium. However, apoptosis remained slightly elevated in these gastric mucosa specimens even three months after eradication. Moreover in GU patients in the present study, bacterial eradication did not decrease the level of apoptosis in the marginal mucosa of the ulcer. These results imply that apoptosis in the gastric mucosa of $H$ pylori infected patients is triggered by both the bacterial products and the host events induced in the mucosa as a result of infection.

The mechanism by which $H$ pylori promotes apoptosis has yet to be elucidated; however, several bacterial products, including urease and vacuolating toxin, and the bacterial load are considered to be mainly responsible. ${ }^{15} 16$ Analysis of these factors showed the urease activity to be closely related to the level of apoptosis in the gastric mucosa. $H$ pylori has a remarkably high urease activity by which it hydrolyses urea to ammonia and carbon dioxide. ${ }^{17}$ As a result, patients with this infection have reduced concentrations of urea and increased concentrations of ammonia in their gastric juice. ${ }^{18}$ Several investigators have shown ammonia to be deleterious to the gastric mucosa, suggesting the importance of urease and ammonia in the pathophysiology of gastroduodenal diseases in $H$ pylori infected patients. ${ }^{819} 20$ Furthermore, Tsuji et al suggested that ammonia plays a crucial role in the induction of apoptosis by demonstrating mucosal atrophy in the stomach after the oral administration of ammonia. They also reported that the damaging stimulus of this treatment is related to the specific action of ammonia, especially non-ionised ammonia, because it can easily penetrate cell membranes and affect the intracellular organelles. Such injury to the epithelial cells is thought to be a trigger for apoptosis in gastric mucosa colonised by $H$ pylori with high urease activity. A more rigorous assessment of the role of urease in apoptosis will require measurement of the extent of apoptosis in an appropriate gastric cell line after incubation with either wild type $H$ pylori or an isogenic urease negative mutant.

Wagner and colleagues ${ }^{21}$ reported that treating gastric cells with tumour necrosis factor $\alpha$, a receptor-activating Fas antibody, and interferon $\gamma$ strongly potentiated $H$ pylori induced apoptosis, suggesting that $H$ pylori indirectly affects the gastric epithelial cells by sensitising them for apoptosis induced by the proinflammatory cytokines in addition to the direct toxic effect of the bacterial products. This finding is comparable with our finding that apoptosis is still higher in DU patients than in non-infected healthy volunteers after the eradication of $H$ pylori. Moreover, in GU patients, the eradication of $H$ pylori did not affect the level of apoptosis at all whereas the level was significantly higher than that of $H$ pylori negative healthy volunteers. Apoptosis induction is thus suggested to be initially triggered by bacterial products, but is soon thereafter dominated by 
other mechanisms such as a cell mediated mechanism in patients predisposed to GU.

In the DU patients analysed in this study, the increase in apoptosis in the antral mucosa correlated closely with the stage of ulceration, suggesting that apoptosis plays an important role in the development of ulceration. On the other hand, neither the size of the bacterial load nor the extent of inflammation in the antral mucosa showed any significant correlation with the DU stage. The augmented apoptosis associated with the development of ulceration thus seems to be induced by some factor other than the bacterial products and mucosal inflammation that accompany infection, even though the $H$ pylori infection itself initiated the series of apoptotic events leading to ulceration.

It is well known that $H$ pylori infection and DU disease are correlated. However, the bacteria mainly colonise the antrum, indicating an indirect pathogenic mechanism. Antral distension was shown to inhibit gastric acid secretion in healthy persons, but this inhibitory mechanism was not observed in patients with DU disease. On the basis of these findings, Olbe and colleagues ${ }^{22}$ hypothesised that $H$ pylori infection of the antrum may selectively block the inhibitory reflex pathways from the antrum to the gastrin and parietal cells, resulting in hypergastrinaemia and increased and prolonged acid secretion as a prerequisite to the development of DU. In fact, they showed that antral distension had no inhibitory effect on gastric acid secretion in $H$ pylori infected patients, but this pathological phenomenon was irrelevant to whether or not they developed DU disease. In the present study, we found apoptosis to be induced in the antrum by infection with $H$ pylori. Moreover, the increase in apoptosis in the antral mucosa correlated significantly with the development of ulceration in the duodenum. It remains to be elucidated whether or not this apoptotic event is related to any abnormalities in the inhibitory reflex pathways from the antrum.

El-Omar and colleagues ${ }^{23}$ reported another mechanism by which $H$ pylori induces DU, although this bacterium predominantly affects the gastric mucosa. They found that $H$ pylori infected patients with DU had a significantly increased basal acid output and an enhanced acid response to exogenous gastrin and gastrinreleasing peptide. Although the abnormality in the basal acid output fully resolved after $H$ pylori eradication, the enhancement of the acid response to gastrin remained unchanged even after the eradication. It therefore seems that the increased acid response in infected patients with DU is not directly induced by the bacterial products but instead by some host mediated event. It thus remains to be eluci- dated whether or not apoptosis is related to an increased acid response in DU patients.

In conclusion, we found apoptosis to be accelerated in the antral mucosa of DU patients infected with $H$ pylori, and urease activity was suggested to be a cardinal causal factor. Moreover, the increase in apoptosis was found to correlate closely with the development of ulceration in the duodenum, suggesting that apoptosis plays a key role in DU disease.

1 Marshall BJ. Helicobacter pylori. Am $\mathcal{f}$ Gastroenterol 1994;89:S1 16-28

2 Lee A, Fox J, Hazell S. Pathogenicity of Helicobacter pylori: a perspective. Infect Immun 1993;61:1601-10.

3 Levi S, Beardshall K, Haddad G, et al. Campylobacter pylori and duodenal ulcers: the gastrin link. Lancet 1989;i: $1167-8$.

4 Graham DY, Opekum A, Lew GM, et al. Helicobacter pylori-associated exaggerated gastrin release in duodenal ulcer patients. The effect of bombesin infusion and urea ingestion. Gastroenterology 1991;100:1571-5.

5 Thompson CB. Apoptosis in the pathogenesis and treatment of disease. Science 1995;267:1456-62.

6 Hall PA, Coates PJ, Ansari A, et al. Regulation of cell number in the mammalian gastrointestinal tract: the importance of apoptosis. F Cell Sci 1994;107:3569-77.

7 Moss SF, Calam J, Agarwal B, et al. Inductin of gastric epithelial apoptosis by Helicobacter pylori. Gut 1996;38:498501.

8 Tsuji M, Kawano S, Tsuji M, et al. Cell kinetics of mucosal atrophy in rat stomach induced by long term administration of ammonia. Gastroenterology 1993;104:796-801.

9 Sakita T, Oguro Y, Takasu S, et al. Observation on the healing of ulceration in early gastric cancer. Gastroenterology 1971;60:835-44.

10 Kabir AMA, Aiba Y, Takagi A, et al. Prevention of Helicobacter pylori infection by lactobacilli in a gnotobiotic murine model. Gut 1997;41:49-55.

11 Mori T, Ando K, Tanaka K, et al. Fas-mediated apoptosis of the hematopoietic progenitor cells in mice infected with murine cytomegarovirus. Blood 1997;89:3565-73.

12 Kobayashi Y, Okazaki K, Murakami K. Adhesion of Helicobacter pylori to gastric epithelial cells in primary cultures obtained from stomachs of various animals. Infect Immun 1993;61:4058-63.

13 Gavrieli Y, Sherman Y, Ben-Sasson SA. Identification of programmed cell death via specific labelling of nuclear programmed cell death via specific labelling of
DNA fragmentation. $\mathcal{F}$ Cell Biol 1992;119:493-501.

14 Kamiya S, Osaki T, Kumada J, et al. Effect of sofalcone on adherence, production of vacuolating toxin, and induction of interleukin- 8 secretion by Helicobacter pylori. $\mathcal{F}$ Clin Gastroenterol 1997;25:S172-8.

15 Smoot DT, Mobley HLT, Chippendale GR, et al. Helicobacter pylori urease activity is toxic to human gastric epithelial cells. Infect Immun 1990;58:1992-4.

16 Atherton JC, Peek Jr RM, Tham KT, et al. Clinical and pathological importance of heterogeneity in vacA, the vacuolating cytotoxin gene of Helicobacter pylori. Gastroenterology 1997;112:92-9.

17 Marshall B, Langton S. Urea hydrolysis in patients with Campylobacter pylori infection. Lancet 1986;i:965-6.

18 Chittajallu RS, Neithercut WD, Macdonald AMI, et al. The effect of increasing Helicobacter pylori ammonia production by urea infusion on plasma gastrin concentrations. Gut tion by urea infusic

19 Murakami M, Saita H, Teramura S, et al. Gastric ammonia has a potent ulcerogenic action on the rat stomach. Gastroenterology 1993;105:1710-15

20 Lichtenberger LM, Dial EJ, Romero JJ, et al. Role of luminal ammonia in the development of gastropathy and hypergastrinemia in the rat. Gastroenterology 1995;108:320-9.

21 Wagner S, Beil W, Westermann J, et al. Regulation of gastric epithelial cell growth by Helicobacter pylori: evidence for a major role of apoptosis. Gastroenterology 1997;113:183647.

22 Olbe L, Hamlet A, Dalenback J, et al. A mechanism by which Helicobacter pylori infection of the antrum contributes to the development of duodenal ulcer. Gastroenterology 1996;110:1386-94.

23 El-Omar EM, Penman ID, Ardill JES, et al. Helicobacter pylori infection and abnormalities of acid secretion in patients with duodenal ulcer disease. Gastroenterology 1995;109:681-91. 